\title{
STITCHING TYPE LARGE APERTURE DEPOLARIZER FOR GAS MONITORING IMAGING SPECTROMETER
}

\author{
Xiaolin Liu*, Ming Li, Ning An, Tingcheng Zhang, Guili Cao, Shaoyuan Cheng \\ Beijing Institute of Space Mechanics \& Electricity, Beijing Key Laboratory of Advanced Optical Remote Sensing \\ Technology, China - (mojiexiaolin, 13521820892, anningji, tczhang1217, bitlitian,csycf) @ 163.com
}

Commission III, WG III/8

KEY WORDS: Polarization, Depolarizer, Muller matrix, Imaging Spectrometer, Stitching, Numerical Analysis

\begin{abstract}
:
To increase the accuracy of radiation measurement for gas monitoring imaging spectrometer, it is necessary to achieve high levels of depolarization of the incoming beam. The preferred method in space instrument is to introduce the depolarizer into the optical system. It is a combination device of birefringence crystal wedges. Limited to the actual diameter of the crystal, the traditional depolarizer cannot be used in the large aperture imaging spectrometer (greater than $100 \mathrm{~mm}$ ). In this paper, a stitching type depolarizer is presented. The design theory and numerical calculation model for dual babinet depolarizer were built. As required radiometric accuracies of the imaging spectrometer with $250 \mathrm{~mm} \times 46 \mathrm{~mm}$ aperture, a stitching type dual babinet depolarizer was design in detail. Based on designing the optimum structural parmeters, the tolerance of wedge angle, refractive index, and central thickness were given. The analysis results show that the maximum residual polarization degree of output light from depolarizer is less than $2 \%$. The design requirements of polarization sensitivity is satisfied.
\end{abstract}

\section{INTRODUCTION}

For earth-observation instrument, changing polarization properties of incoming beam can either be the property to detect or a performance limiting factor. In the imaging spectrometer used for greenhouse gases monitoring, the grating is chosen as dispersion element, which has a variable efficiency with respect to the polarization state of the beam. Moreover, the accuracy performance required for these instruments continues to increase, and a relative accuracy is less than $0.1 \%$. At the same time, the polarization state of the incident beam is unknown in advance. Thus, the imaging spectrometer must strive to achieve low polarization sensitivity. Using the gratings with low polarization sensitivity is an obvious solution. However, achieving such low polarization sensitivities may require a trade off with diffraction efficiency. For large bandwidths, it is also exceedingly difficult or impossible to achieve such low polarization sensitivities. Therefore, the demand arises for a device that would depolarize the incoming beam, to be included before the most polarizing optical element or system.

The common device to depolarization is a so-called depolarizer, which is a combination of birefringence crystals with certain wedge. The study of depolarizer has been developed for thirty years. Recently, the types of depolarizer include double wedge H-V depolarizer (Stephen et al., 1992; Zhao et al., 2011), double wedge optically active depolarizer (Song and Song, 2009), Lyot depolarizer (Waluschka et al., 2011; Burns, 1983), and dual Babinet depolarizer(McGuire and Chipman, 1990). Dual Babinet depolarizer has many advantages. It has depolarization effect for all polarized light, and is independent on the thickness of crystals. It is widely used in high-precision atmospheric detection (Dobber et al., 2005).

The aperture of the crystal is limited to the actual manufacturing capacity, and the largest diameter of crystal growth is less than $100 \mathrm{~mm}$. For large aperture imaging spectrometer, the traditional depolarizer is not applicable exceedingly. Therefore, the need is presented for new structure to achieve large aperture depolarizer.

In this paper, we present a novel structure by stitching method for large size depolarizer based on dual Babinet depolarizer. We design the novel depolarizer for a certain greenhouse gas imaging spectrometer. The design theory and numerical calculation model for stitching type depolarizer is built, according to the polarized and crystal optics. The depolarization effect is researched with respect to different factors including wedge angle, refractive index and diameter.

\section{STITCHING STRUCTURE AND THEORY}

Dual Babinet depolarizer is consisted of four wedges of birefringent material arranged in pairs, as shown in Figure 1. The wedges of a pair are bonded with crossed crystal axes, and the first pair is rotated by $45^{\circ}$ w.r.t. the second one. Light beams passing through this device experience polarization dependent phase delays, which vary over the pupil and the resulting polarization states at the exit largely average out.

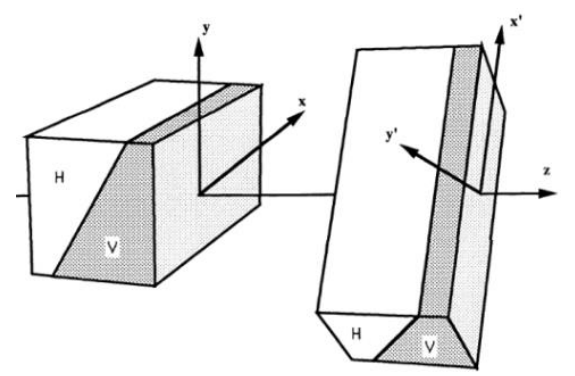

Figure 1. Structure of the dual Babinet depolarizer

*mojiexiaolin@163.com 
Dual Babinet depolarizer is made of quartz generally. The ideal position for the scrambler would be in front of the telescope so that all optical components are illuminated with depolarized light. For large aperture imaging spectrometer, the diameter of depolarizer must be increased with the pupil diameter. However, the largest diameter of the crystal growth is less than about $100 \mathrm{~mm}$. A stitching type dual Babinet depolarizer was presented in this paper. Several sets of dual Babinet structures are juxtaposed instead of one whole, as shown in Figure 2. This method greatly reduces the diameter of the crystal, and achieves the large aperture depolarization effect at the same time. Mechanical structure is used between each set. In addition, to enhance reliability of the space environment, here we use air gap instead of traditional gluing method between these wedges.

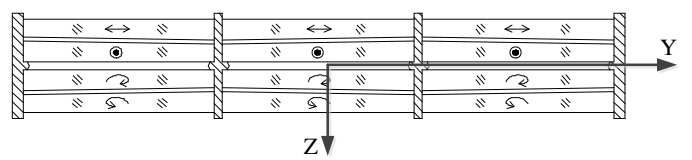

Figure 2. Structure parameter of the dual Babinet depolarizer

Stitching type dual Babinet depolarizer is equal to divide the entire aperture into several sub-apertures. The output beam is made of a mix of polarization states, and the system will be polarization insensitive because of the averaging effect of the pupil. The calculation method of degree of depolarization for Stitching type dual Babinet depolarizer is different from the conventional structures, and the depolarization character of stitching type dual Babinet depolarizer is also not equivalent to a whole aperture depolarizer. In this paper, the calculation method is built for Stitching type dual Babinet depolarizer.

The degree of depolarization is calculated using Mueller algebra. To simplify the computational model, the following small effects are not treated in this calculation: (1) the refraction effects at interfaces and (2) diattenuation from the differing absorption coefficients for the extraordinary and ordinary rays. The coordinate is shown in Figure 2, the light normally incidence the depolarizer from the top surface.

In one dual Babinet depolarizer, the wedges of each pair are bonded with crossed crystal axes, and can be is respectively equivalent to a linear retarder oriented at 0 degree and 45 degree. For simplicity, we choose the wedges for the dual Babinet depolarizer to have identical central thickness and slopes. The retardance between the ordinary and extraordinary light is:

$$
\varphi(y)=\frac{4 \pi}{\lambda} \Delta n(\lambda) y \tan \alpha=\delta y
$$

Where $\Delta n(\lambda)=n_{e}(\lambda)-n_{o}(\lambda), n_{o}(\lambda), n_{e}(\lambda)$ are theordinary and extraordinary indices of refraction, $\alpha$ is wedge angle, $\delta$ is the slope of the retardance variation. Quartz is usually choosed as the crystalline material for depolarizer. The retardance of a Babinet compensator varies linearly with position $y$.

The the Mueller matrix for $0^{\circ}$ Babinet compensator (first pair) is

$$
\mathbf{M}_{0}=\left[\begin{array}{cccc}
1 & 0 & 0 & 0 \\
0 & 1 & 0 & 0 \\
0 & 0 & \cos \delta y & \sin \delta y \\
0 & 0 & -\sin \delta y & \cos \delta y
\end{array}\right],
$$

The Mueller matrices for Babinet compensators oriented at $45^{\circ}$ is:

$$
\mathbf{M}_{45}=\mathbf{R}(45) \mathbf{M}_{0} \mathbf{R}(-45)=\left[\begin{array}{cccc}
1 & 0 & 0 & 0 \\
0 & \cos \delta y^{\prime} & 0 & \sin \delta y^{\prime} \\
0 & 0 & 1 & 0 \\
0 & -\sin \delta y^{\prime} & 0 & \cos \delta y^{\prime}
\end{array}\right]
$$

where, $\mathbf{R}(\alpha)$ is the rotation matrix, the ${ }^{y}$ coordinate system has been rotated from y by $45^{\circ}$. Multiplying Eq. (3) and Eq. (2) gives the spatially varying Mueller matrix for the dual Babinet depolarizer.

$$
\begin{aligned}
& \mathbf{M}_{\mathrm{DB}}=\mathbf{M}_{45} \mathbf{M}_{0} \\
& =\left[\begin{array}{cccc}
1 & 0 & 0 & 0 \\
0 & \cos \delta y^{\prime} & -\sin \delta y \sin \delta y^{\prime} & \sin \delta y \cos \delta y^{\prime} \\
0 & 0 & \cos \delta y & \sin \delta y \\
0 & -\sin \delta y^{\prime} & -\cos \delta y^{\prime} \sin \delta y & \cos \delta y \cos \delta y^{\prime}
\end{array}\right]
\end{aligned}
$$

The stokes vector of the light output is

$$
\begin{aligned}
& \mathbf{S}_{o}=\mathbf{M}_{D B} \mathbf{S}_{i} \\
& =\left[\begin{array}{c}
1 \\
\cos \delta y^{\prime} \cos 2 \varphi-\sin \delta y \sin \delta y^{\prime} \sin 2 \varphi \\
\cos \delta y \sin 2 \varphi \\
-\sin \delta y^{\prime} \cos 2 \varphi-\cos \delta y^{\prime} \sin \delta y \sin 2 \varphi
\end{array}\right]
\end{aligned}
$$

Where, $\mathbf{S}_{i}$ is the incident line polarized light:

$$
\mathbf{S}_{i}=\left[\begin{array}{llll}
1 & \cos 2 \varphi & \sin 2 \varphi & 0
\end{array}\right]^{\mathrm{T}}
$$

The average stokes vector of exit rays should be integral along the pupil S:

$$
\overline{\mathbf{S}}_{o}=\left[\begin{array}{l}
A \\
B \\
C \\
D
\end{array}\right]=\left[\begin{array}{c}
\iint_{s} 1 d x d y \\
\iint_{s} \cos \delta y^{\prime} \cos 2 \varphi-\sin \delta y \sin \delta y^{\prime} \sin 2 \varphi d x d y \\
\sin 2 \varphi \iint_{s} \cos \delta y d x d y \\
\iint_{s}-\sin \delta y^{\prime} \cos 2 \varphi-\cos \delta y^{\prime} \sin \delta y \sin 2 \varphi d x d y
\end{array}\right]
$$

For traditional depolarizer, the pupil area is continuous, thus the integral operation is relatively simple. For Stitching type dual depolarizers, the coordinate system must be respectively established for each area, and independent integral at each area is carried out to get each average stokes vector by Eq. (7). Therefore, Eq. (7) is rewritten as:

$$
\overline{\mathbf{S}}_{o}^{\prime}=\left[\begin{array}{c}
A^{\prime} \\
B^{\prime} \\
C^{\prime} \\
D^{\prime}
\end{array}\right]=\left[\begin{array}{c}
A_{\Sigma 1}+A_{\Sigma 2}+A_{\Sigma 3} \\
B_{\Sigma 1}+B_{\Sigma 2}+B_{\Sigma 3} \\
C_{\Sigma 1}+C_{\Sigma 2}+C_{\Sigma 3} \\
D_{\Sigma 1}+D_{\Sigma 2}+D_{\Sigma 3}
\end{array}\right]
$$

Where, $\Sigma_{1}+\Sigma_{2}+\Sigma_{3}$ is the sum of three stitching areas along pupil, and $A_{\Sigma i}, B_{\Sigma i}, C_{\Sigma i}, D_{\Sigma i}$ are the elements of average stokes vector of Area $i$. 
The expression of the residual polarization is:

$$
P=\sqrt{A^{2}+B^{\prime 2}+C^{\prime 2}} / D^{2}
$$

To make P near to zero, $B^{\prime}, C^{\prime}$, and $D^{\prime}$ should be near to zero.

According to the Eq. (1), Eq. (7), Eq. (8), Eq. (9), the residual polarization is a function of wavelength, wedge angle and aperture size, and independent on the central thickness. The wavelength and aperture size is determined by the spectrometer, and wedge angle is the sole parameters which can be optimized for the design of depolarizer.

\section{DEPOLARIZER DESIGN}

A gas monitoring imaging spectrometer is used for testing $\mathrm{CO}_{2}$ concentration. The spectral region is $1591 \sim 1621 \mathrm{~nm}$, the focal length in spatial dimension is $328 \mathrm{~mm}$, and the spectral resolution is $0.07 \mathrm{~nm}$. The pixel size of detector is $32 \mu \mathrm{m}$, and 28 pixels are combined into a pixel using binning technology. Its pupil aperture is $250 \mathrm{~mm} \times 46 \mathrm{~mm}$ 。 The requirement of residual polarization sensitivity of depolarizer would be below $2 \%$ in design stage.

The ideal position for the depolarizer would be in front of the telescope so that all optical components are illuminated with depolarized light. The material of the depolarizer is quartz. However, limited to the actual diameter of the quartz crystal, it cannot be implemented by a whole crystal, because the aperture is $250 \mathrm{~mm} \times 46 \mathrm{~mm}$. Here we use sub-aperture stitching method as shown in Figure 3. Three sets of dual Babinet structures are juxtaposed instead of one whole. The cross sectional dimensions are labelled in Figure 3. The width of stitching area is $2 \mathrm{~mm}$.

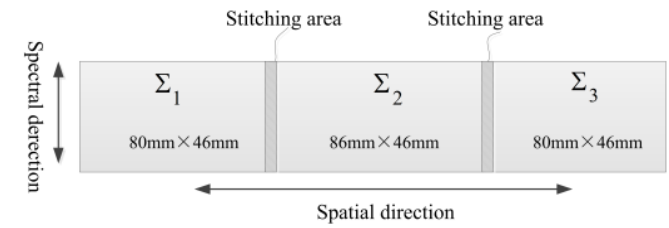

Figure 3. Schematic diagram of sub-aperture stitching of depolarizer

The Eq. (7) is too complicated, especially for rectangular pupil, and the residual polarization can't get the analytic solution. However, it can be analyzed with the numerical solution, and we can deduce the relationship of residual polarization with the wedge angle.

According to the Eq. (1), Eq. (7), Eq. (8), Eq. (9), the polarization degree of output light with different wedge angle and incident polarized light have been simulated analysis under the central wavelength $1606 \mathrm{~nm}$. The calculated results are shown in Figure 4.

As shown in Figure 4, the maximum residual polarization sensitivity is changed with the polarization direction of incident light and reaches the peak point at odd times of $\pi / 4$. Therefore, we only analyse the output polarization degree at $45^{\circ}$ polarized light incident, to evaluate the maximum residual polarization sensitivity of depolarizer.

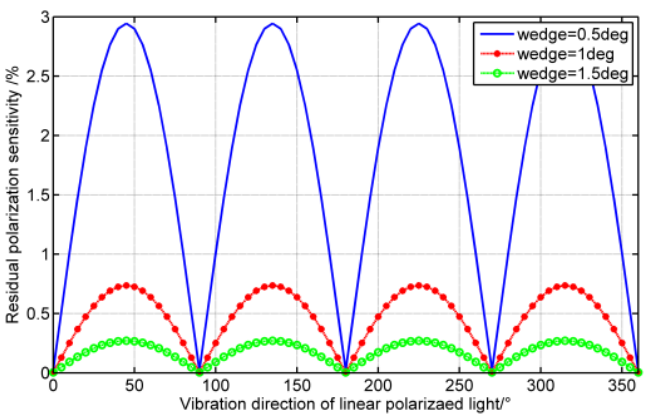

Figure 4. Polarization degree of output light from depolarizer changed with the incident polarization at $1606 \mathrm{~nm}$

The numerical solve of residual polarization for different wavelengths and different wedge angle is displayed in Figure 5. The residual polarization is changed with the two independent variables respectively, the wavelengths and wedge angle. As the wedge angle increasing, the residual polarization decreased with fluctuation. The reason of the oscillation is the integral results of $B, C, D$ in Eq.(7), which is a function of variation in retardance $\varphi(y)$ in Eq.(1). As the wavelength increasing, the magnitude is bigger and the speed is slower. Therefore, once the residual polarization of the depolarizer at the longest wavelength is in the required range, it also satisfies the requirement for the whole band range.

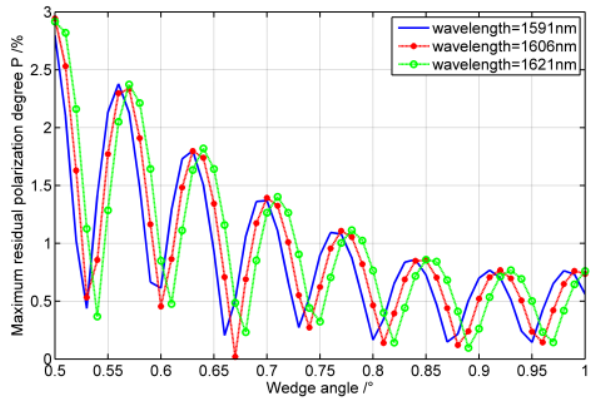

Figure 5. The maximum residual polarization sensitivity changed with wedge angle

A known feature of Dual Babinet depolarizer is that polarized light passing through the four wedges is split into four beams due to birefringence. This effect is cannot be avoided and reduces imaging performance. Therefore, the wedge angle cannot be infinite, and have to consider the balance between depolarization characteristics and imaging quality. As shown in Figure 5, the maximum residual polarization sensitivity is less than $2 \%$, when the wedge angle is greater than $0.72^{\circ}$.

\section{DISCUSSION}

In this section, the traditional full aperture structure (Structure A) and stitching aperture structure proposed in this paper (Structure B) are compared and analysed. The maximum residual polarization sensitivity of full aperture structure is simulated analysis, as shown in Figure 6. Its aperture is $250 \mathrm{~mm} \times 46 \mathrm{~mm}$, which is the same as the total aperture of Structure B in section 3 . 


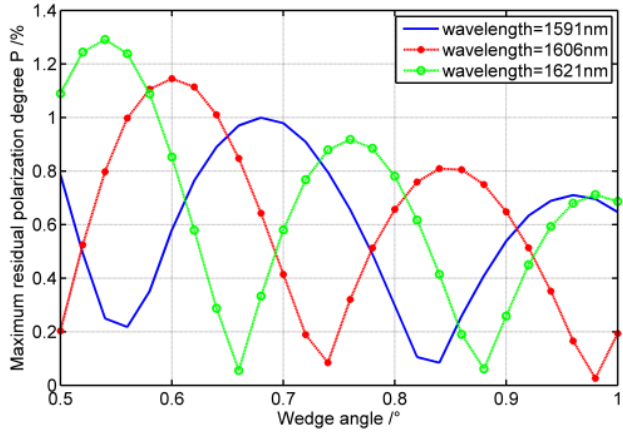

Figure 6 . The maximum residual polarization sensitivity changed with wedge angle for full aperture structure

Here we compare the depolarization effect of Structure A illustrated in Figure 6 and the Structure B with stitching method illustrated in Figure 5. As the wedge angle increasing, the residual polarization of Structure $\mathrm{B}$ decreased faster than Structure A, although the magnitude is bigger at the initial phase. The maximum residual polarization is both less than $0.8 \%$, when the wedge angle is greater than 0.8 . Therefore, Structure B is not inferior to Structure B on depolarization effect. The key is Structure B solve the problem of large aperture depolarization by stitching method. The cost of manufacturing is also greatly reduced.

\section{CONCLUSION}

A novel structure by stitching method for large size depolarizer based on dual Babinet depolarizer is presented. The design theory and numerical calculation model for stitching type depolarizer is built. As required radiometric accuracies of the imaging spectrometer with $250 \mathrm{~mm} \times 46 \mathrm{~mm}$ aperture, a stitching type dual babinet depolarizer was design in detail. The analysis results show that the maximum residual polarization degree of output light from depolarizer was less than $2 \%$, when the wedge angle is greater than $0.72^{\circ}$. This method greatly reduces the diameter of the crystal, and achieves the large aperture depolarization effect.

\section{ACKNOWLEDGEMNTS}

The work was supported by the National Key Research and Development program of China (No.2016YFB0500802).

\section{REFERENCES}

Stephen C.M, Peter W.Mn, Chipman R.A, 1992. Design and analysis of a depolarizer for the NASA moderate-resolution Imaging Spectrometer-Tilt. SPIE, 1746:375-385

Zhao F. C,Wang S.R, Qu Y, et al., 2011. Design and analysis of a depolarizer for the space-borne grating imaging spectrometer. Spectroscopy and Spectral Analysis, 31(7):1991-1994.

Song S.X, Song L.K, 2009. Analysis of double plate rotation depolarizer by mueller matrix. Acta Optica Sinica, 29(7): 19471950 .

Waluschka E, Wilson M, Quijada M, et al., 2011. ORCA's depolarizer. SPIE, 8153: 81530U.
Burns W.K, 1983. Degree of polarization in the Lyot depolarizer. Journal of Lightwave Technology, 1(3):475-479.

McGuire J, Chipman R.A, 1990. Analysis of spatial pseudodepolarizers in imaging systems. Optical Engineering, 29(12):1478-1484 